\title{
Commentary
}

\section{Laser treatment of soft drusen in age-related maculopathy}

The disappointing results of treatment of choroidal neovascularisation (CNV) in age-related maculopathy (ARM) is focusing increasing attention on prevention in eyes at high risk. Clinical and histological studies have consistently identified the presence of soft drusen as a risk factor for the development of CNV in ARM. The observation that photocoagulation can cause drusen to regress therefore suggests that prophylactic laser treatment might delay the complications associated with high risk drusen. Several prospective trials of prophylactic laser have already been undertaken $^{1-4}$ and more are planned, but the standard questions remain to be answered: is it effective, is it safe, and is it worthwhile?

Is it effective? Results of treatment might be judged in the context of case selection and technique used. At present there is no agreement on the number of burns, on whether to apply a temporal horseshoe $e^{1-3}$ or a ring, ${ }^{45}$ or on whether drusen should be treated directly. Moreover, not all soft drusen carry the same risk. In those over the age of 65 soft drusen develop as part of a diffuse deposition of coiled membranes (basal linear deposit). These drusen coincide with the period of high risk of $\mathrm{CNV}$, even though they are often smaller than $250 \mu \mathrm{m}$ and shallow. Other soft drusen, however, originate from breakdown of hard drusen, while deposits over $500 \mu \mathrm{m}$ in size appear to have, in addition, a fluid component. These latter types, which occur also in younger patients, differ from the membranous type in that they may remain a focal pathology and lead to only a localised patch of atrophy.

The mechanism whereby laser induces the regression of both local and remote drusen is unclear. Direct treatment to drusen may accelerate their removal by phagocytes and, as a result of damage to the retinal pigment epithelium (RPE), less debris will be produced. However, why laser treatment also has an indirect effect causing regression of drusen within the fovea is less clear. It has been postulated that lipids accumulating in Bruch's membrane impair the transfer of fluid out of the retina and laser may alter the physical characteristics of the lipids in a way that allows the escape of fluid drusen material. This is supported by the speed and completeness with which the larger more fluid drusen resorb.

Histological studies of senile postmortem eyes commonly demonstrate small clinically unsuspected new vessels, especially if $\mathrm{CNV}$ is present in the fellow eye, so how is the risk of CNV reduced by causing soft drusen to regress? Having penetrated the collagenous layers of Bruch's membrane, new vessels grow initially in the cleavage plane created by drusen and, since the laser burns cause this debris to clear with resulting adhesion of the RPE to Bruch's membrane, a barrier would be created. The beneficial effect of laser may therefore be the limitation of spread rather than the prevention of neovascularisation.

Is it safe? The paramount safety consideration is the risk of inducing CNV. Caution is advised in eyes with hyperpigmentation since this increases the risk of developing $\mathrm{CNV}$ in eyes with drusen. Pigment clumping infers that ARM is advanced and that macrophages will already be mobilised beneath Bruch's membrane. Macrophages have been observed in relation to thinned segments in the membrane and this is associated with activation of the underlying choroidal capillaries. By contributing to the amount of debris to be removed, laser may accentuate the macrophage response and the release of angiogenic factors. Fortunately, CNV has been uncommon and, if it should develop at the site of a burn, commences outside the fovea where it may be suited to photoablation. Moreover, when it has occurred it has mostly been delayed for 6 months or more after the prophylactic laser, by which time considerable clearing of drusen may have taken place and this should discourage rapid spread.

The other possible complication is the development of geographic atrophy of the RPE resulting from expansion of the laser burns. Since degeneration of the RPE is more advanced over drusen, particularly if pigment clumping is already evident, laser applied directly to drusen would hasten atrophy. However, if gentle burns are applied to intervening retina this may not occur and would, in any event, develop in the parafovea, mimicking the natural evolution of geographic atrophy that occurs in the absence of drusen, and so sparing the centre of the fovea.

Is it worthwhile? It has to be remembered that such eyes are not normal and may in time lose vision from drusen related atrophy even if $\mathrm{CNV}$ can be prevented. Nevertheless, this course could delay visual loss for many years. There is even the possibility of delaying atrophy if large fluid foveal drusen are caused to regress before irreversible changes in the RPE develop. Furthermore, many of these patients notice an improvement in visual acuity after treatment.

Long term prospective trials are required on groups of patients selected according to age, drusen type, hyperpigmentation, and presence of $\mathrm{CNV}$ in the fellow eye. Only then can it be determined whether laser induced regression of soft drusen can lower the incidence of $\mathrm{CNV}$, with the possible additional benefits of slowing the development of atrophy and improving visual acuity.

15 Parnell Street, Strathfield,

SHIRLEY SARKS NSW 2135, Australia

1 Sigelman J. Foveal drusen resorption one year after perifoveal laser photocoagulation. Ophthalmology 1991; 98: 1379-83.

2 Figueroa MS, Regueras A, Bertrand J. Laser photocoagulation to treat macular soft drusen in age-related macular degeneration. Retina 1994; 14: 391-6.

3 Frennesson IC, Nilsson SEG. Effects of argon (green) laser treatment of soft drusen in early age-related maculopathy: a 6 month prospective study. Br f Ophthalmol 1995; 79: 905-9.

4 Alió JL, Ruiz-Moreno JM. Evolution of soft drusen maculopathy after focal photocoagulation of the drusen. 4th International Symposium on Ocular Circulation and Neovascularization, 22-26 May 1995, Budapest, Hungary.

5 Sarks SH, Sarks JP, Arnold JA, Gillies MC, Walter CJ. Prophylactic perifoveal laser treatment of soft drusen. Aust NZ $\mathcal{J}$ Ophthalmol 1996 (in press). 